Bulletin d'Histoire Contemporaine de l'Espagne

$50 \mid 2016$

Les intellectuels en Espagne, de la dictature à la démocratie (1939-1986)

\title{
Hermano Lobo (1972-1976) : une revue satirique à la fin de la dictature franquiste
}

\section{Caroline Couvert}

\section{(2) OpenEdition \\ Journals}

Édition électronique

URL : http://journals.openedition.org/bhce/912

DOI : 10.4000/bhce. 912

ISSN : 1968-3723

Éditeur

Presses Universitaires de Provence

Édition imprimée

Date de publication : 1 décembre 2016

Pagination : $277-279$

ISSN : 0987-4135

Référence électronique

Caroline Couvert, «Hermano Lobo (1972-1976) : une revue satirique à la fin de la dictature franquiste», Bulletin d'Histoire Contemporaine de l'Espagne [En ligne], 50 | 2016, mis en ligne le 09 octobre 2018 consulté le 24 septembre 2020. URL : http://journals.openedition.org/bhce/912 ; DOI : https://doi.org/ 10.4000/bhce.912 
Hermano Lobo (1972-1976): une revue satirique à la fin de la dictature franquiste ${ }^{1}$

\section{Caroline COUVERT}

Revue satirique publiée entre 1972 et 1976, Hermano Lobo, apparaît au moment où une partie de la société, impressionnée par l'accession à la démocratie de la Grèce (1973) et du Portugal (1974) aspire à un régime démocratique et souhaite une adhésion du pays à la Communauté économique européenne. À cette époque, la presse est surveillée, bien que la censure ait été assouplie par l'instauration de la loi Fraga en 1966. La presse satirique doit donc composer avec celle-ci en adoptant souvent un langage second. Hermano Lobo représente cette tradition du non-dit et de l'évocation métaphorique, caractéristique de la période dictatoriale. Le travail veut appréhender les enjeux esthétiques et politiques de cette revue satirique à un moment clé de l'histoire contemporaine de l'Espagne.

La question essentielle qui se pose est de savoir comment une telle revue a pu voir le jour et surtout se maintenir au cours des dernières années de la dictature franquiste; mais on ne peut en éluder une autre : pourquoi disparait-elle au début de la Transition lorsque semblent triompher les idées qu'elle défendait et que la presse recouvre la liberté d'expression?

Cette thèse examine d'abord le contexte général de la presse satirique espagnole, les conditions de production définies par la

1 Thèse d'Études Ibériques, dirigée par le prof. Paul Aubert, et soutenue à l'université d'AixMarseille, le 18 novembre 2013. Jury: Mmes et MM. les Prof. Marie Franco (université de Paris III), présidente, Pilar Martínez Vasseur (université de Nantes), Elisabel Larriba (université d'Aix-Marseille), Isabelle Renaudet (université d'Aix-Marseille), Alicia Alted (UNED, Madrid) et Paul Aubert (université d'Aix-Marseille). législation en vigueur, avant de présenter la publication elle-même: structures, journalistes, collaborateurs. Mais au-delà de la dénonciation politique et de la remise en question inspirée par la philosophie (entre François d'Assises et Hobbes) Hermano Lobo veut régénérer la presse humoristique espagnole. Entre tradition et modernité, les créateurs de la revue veulent faire un journal où le graphisme aura une place prépondérante, où la simplicité et l'attractivité répondront à un contexte morose dans l'attente d'une rupture politique. La revue évolue pourtant vers une mise en page plus chargée, une tonalité moins enjouée et un scepticisme inévitable, face à la lenteur du processus démocratique.

Une deuxième partie est consacrée à l'écriture et à la question des procédés humoristiques utilisés aussi bien dans les textes que dans le dessin. Après l'exploration conjointe de l'écriture humoristique et de l'humour graphique, ce sont des notions complexes comme la satire ou la parodie qui sont abordées. Enfin est examinée l'application de ce parti pris humoristique à l'actualité politique nationale et internationale ainsi que la mise en question des valeurs sociales et idéologiques du régime, tout en ouvrant une interrogation récurrente sur l'avenir.

Une troisième partie étudie la thématique et le style des collaborateurs les plus réguliers de la revue, Manuel Vicent, Francisco Umbral et Manuel Vázquez Montalbán.

Plusieurs axes ont été suivis : l'analyse du discours de la revue (et notamment celle de la relation entre le texte et le graphisme), l'étude du rôle et de la portée de celui-ci au sein de la société espagnole, ainsi que l'étude de l'évolution de la revue, sa place au sein de la presse satirique anti-franquiste, ainsi que les raisons de sa disparition.

Pour éviter la censure, trois tonalités étaient utilisées: l'humour, la satire et l'ironie. L'humour servait à présenter de 
façon légère des opinions sur des affaires souvent graves. Comparer le mariage à un suicide permettait de faire rire tout en insinuant que le divorce pouvait être parfois une solution. Faire des jeux de mots sur la participation ou la droite et la gauche, grâce à leur polysémie, favorisait la transmission de messages politiques d'une façon anodine. L'ironie, elle, permettait de dire le contraire de ce que l'on pensait, en faisant croire de façon dérisoire que l'on approuvait la politique du régime jusque dans ses préceptes les plus ridicules. Enfin la satire dénonçait l'absurdité d'une situation de façon plus acerbe et vindicative. Ces trois points de vue se trouvent réunis dans les textes et les dessins. Cependant l'humour graphique a été le plus important et sans doute le plus efficace.

La revue eut recours à la reprise de quelques leitmotivs qui montraient la volonté des journalistes de fidéliser leur lectorat, mais finalement au moment de la disparition du régime franquiste, ils ont sans doute contribué à l'essoufflement de la revue qui avait voulu réconcilier le peuple avec la politique. Car la revue (dont le référent explicite était son homologue française Charlie Hebdo) qui disparaît en juin 1976, avait ouvert la voie à un nouveau genre de presse humoristique, plus en adéquation avec son temps, telles que Barrabás, El Papus ou Por favor.

Hermano Lobo dénonce la politique du franquisme tardif et son anachronisme au sein d'une Europe Communautaire. Son originalité et son succès dépendent alors d'une série procédés stylistiques, rhétoriques et thématiques. Des figures classiques comme la métaphore, la comparaison ou le comique de mots, jusqu'aux allégories et métonymies les plus accusatrices, les journalistes revendiquent leur opposition à la dictature par le biais de l'humour. Mais la revue a recours également à une thématique précise. Le détour par l'étranger acquiert ainsi dans ses pages une dimension critique : dénoncer la guerre du Vietnam, c'est dénoncer l'acharnement anticommuniste; critiquer Nixon dans l'affaire du Watergate, c'est démontrer que finalement, Franco, qui bannit le pluralisme politique, évite ce genre de scandale; évoquer la Révolution des aillets au Portugal, c'est montrer qu'une dictature vieillissante, et en contradiction avec l'évolution européenne, peut être renversée du jour au lendemain, sans heurts. Enfin, parler de la Communauté Économique Européenne, c'est rappeler l'exclusion de l'Espagne qui, de par son régime politique, ne correspond pas aux prérogatives des États membres.

Un autre thème important est celui de la libéralisation des mœurs et de la femme. $\mathrm{La}$ revue revendique le progrès social et se réfère constamment à l'ouverture au tourisme qui reflètent la réalité des années soixante-dix en Espagne. Grâce à ce parallélisme les journalistes dénoncent l'immobilisme politique. La femme devient donc l'allégorie de la politique : la libéralisation, l'adultère, sont brandis pour narguer une dictature qui s'enlise et qui refuse le pluralisme et les courants libéraux européens. Enfin, des leitmotivs politiques vont venir s'ajouter à la thématique précédemment évoquée : le parti unique et les associations, la répression et l'amnistie, la censure et la condition de la presse. Les journalistes, à force de répétition, veulent ancrer dans l'esprit des lecteurs l'image d'un pays politiquement désuet, freiné dans ses négociations économiques par une dictature qui perdure. Ces leitmotivs ont fonctionné de façon cyclique, s'éteignant d'eux-mêmes lorsque le sujet n'avait plus lieu d'être.

C'est ainsi que le " frère loup » contre lequel s'étaient battus les journalistes disparaissait au profit d'une ouverture politique qui, même si elle n'était pas suffisamment radicale selon les partisans de la rupture démocratique, rendait les propos de Hermano Lobo obsolètes. II 
fallait s'adapter à une situation nouvelle, où l'information quotidienne, le pragmatisme et l'oubli d'une division entre vainqueurs et vaincus devenaient essentiels pour les Espagnols. La revue s'éteint finalement à la veille de la Loi pour la Réforme politique instaurée par le gouvernement Suárez. Néanmoins, Hermano Lobo reste aujourd'hui un témoignage de ce qu'a pu être le combat de la presse à la fin de la dictature franquiste ainsi que le symbole d'une opposition muselée. Elle représente le refuge idéologique de ceux qui réclamaient une liberté d'expression, le théâtre dans lequel les revendications étaient exprimées à demi-mot, dans la limite autorisée.

Avec des auteurs de talent comme Manuel Vicent et Francisco Umbral, et des humoristes graphiques comme Manuel Summers, Chumy Chúmez ou El Perich, Hermano Lobo a ainsi pour objectif de dénoncer la dictature. Pour cela, elle choisit de baser son discours sur la démonstration, l'enseignement et la réitération, dans une optique pédagogique. Elle veille à (ré)inculquer aux citoyens un intérêt pour la situation politique de leur pays. En insérant un vocabulaire démocratique elle lutte en faveur d'une rupture à laquelle elle n'assistera finalement pas. Grâce à ces codes langagiers mis en place, les journalistes montrent alors que l'immobilisme de la dictature ne correspond plus à une société en pleine mutation. Les talents de Vicent, Umbral et Vázquez Montalbán qui envisagent trois stratégies différentes, vont s'avérer complémentaires. La conjugaison de la pédagogie à tout prix (Vicent), de l'ironie de la fable (le personnage de Maripi, pour Umbral) et d'une hésitation entre le scepticisme et la fiction (le personnage d'Encarna chez Vázquez Montalbán) concourent à l'émergence d'un discours public.

Hermano Lobo reste l'incarnation d'un projet qui a rassemblé des journalistes, des dessinateurs et des intellectuels dont l'inspiration et le talent ont permis de faire rire les lecteurs et d'observer la situation de l'Espagne avec un détachement souvent teinté d'amertume, en attendant l'arrivée de la démocratie. Il ne fait aucun doute qu' Hermano Lobo a contribué à l'apparition d'une préoccupation civique sinon à la constitution d'une opinion publique.

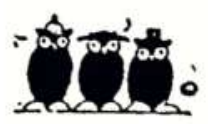

\section{Los nosotros en la Transición: memoria e identidad en las cuatro principales culturas políticas del País Vasco (1975-1980) ${ }^{1}$}

\section{Eider LANDABEREA ABAD}

La sociedad vasca del siglo XXI, plural y diversa en cuanto a sentimientos de pertenencia, ha sido el punto de partida de esta tesis doctoral, que ha tenido como objeto de análisis las memorias de las cuatro culturas políticas más representativas de escenario vasco durante la Transición, es decir, el nacionalismo histórico (representado por Euzko Alderdi Jeltzalea-Partido Nacionalista Vasco), la izquierda estatal (representada por el Partido Socialista de Euskadi-Partido Socialista Obrero Español), el centro derecha español (representado por Unión de Centro Democrático) y la izquierda

1 Tesis doctoral defendida el 13 de diciembre de 2013 en la Facultad de Ciencias Sociales y Humanas de la Universidad de Deusto en el campus de Donostia-San Sebastián. Directores: Elena Barrena Osoro y José Antonio Rodriguez Ranz. Tribunal: Presidente: José Luis de la Granja Sainz (Universidad del Pais Vasco). Secretaria: Leyre Arrieta Alberdi (Universidad de Deusto). Vocales: Santiago de Pablo Contreras (Universidad del País Vasco), Josu Chueca Intxusta (Universidad del Pais Vasco) y Santiago Larrazabal Basañez (Universidad de Deusto). 